\title{
Periodic optical variability of AGN
}

\author{
E. Bon ${ }^{1}$, P. Marziani ${ }^{2}$, N. Bon ${ }^{1}$ \\ ${ }^{1}$ Belgrade Observatory, Serbia \\ ${ }^{2}$ INAF, Osservatorio Astronomico di Padova, Italy \\ email: ebon@aob.rs
}

\begin{abstract}
Here we present the evidence for periodicity of an optical emission detected in several AGN. Significant periodicity is found in light curves and radial velocity curves. We discuss possible mechanisms that could produce such periodic variability and their implications. The results are consistent with possible detection of the orbital motion in proximity of the AGN central supermassive black holes.
\end{abstract}

Keywords. quasars: general, black hole physics, accretion, accretion disks

\section{Introduction}

Different mechanisms have been proposed to explain active galactic nuclei (AGN) emission variability (see e.g. Gaskell 1983, 2009 and the references therein). Besides outflows, jet precession, disk precession, disk warping, spiral arms, flares, and other instabilities, one of the most interesting possibilities involves the existence of a binary system in the core (see eg., Komossa 2006; Bogdanović et al. 2008; Gaskell 2009; Bon et al. 2012; Bogdanović 2015; Graham et al. 2015a, 2015b; Bon et al. 16, Charsi et al. 2016, Liu et al. 2016. and the references therein) and the tidal disruption event (see for e.g. Komossa and Zensus 2015). If black hole (BH) mass grows via major mergers we might expect to see the signature of a binary black hole in some or many active galaxies, if the merger process involves slow coalescence of the two components.

The variability in the nuclei of galaxies was recognized even before AGN were defined as a class. There are light curves showing variability in over 100 years of observations in some objects, for example NGC4151 (from 1906, see e.g., Oknyanskij 2007)), 3c273 (from 1880s Smith \& Hoffleit 1963), with long variability timescale of more than one decade. In the 1950 s it was reported that the NGC 5548 nucleus appeared to vary by about a magnitude (see more in Gaskell \& Klimek 2003).

It is well known that many AGN show variability in different time scales and all wavelengths (Gaskell \& Klimek 2003). The variability timescales are affected by speed at which physical processes propagate; most relevant are the speed of light $\mathrm{c} \sim 3 \cdot 10^{5} \mathrm{~km}$ $\mathrm{s}^{-1}$, the orbital speed $v_{\text {orb }} \sim(G M / R)^{1 / 2}$ and the sound speed $v_{s} \sim(k T / m)^{1 / 2}$, where $c>v_{\text {orb }}>>v_{s}$. The shortest timescale corresponds to the light crossing timescale. Reverberation campaigns are based on measuring light travel time between a spectral feature representative of the ionizing continuum and an emission line. If the time delays are measured as a function of radial velocity, it is possible at least in principle to map the line emitting region.

Orbital timescales are longer (Netzer 2013). Measuring them could be very useful for determining the dynamical mass of the central BH, while sound speed timescale can give us information about the property of the disk or the broad line region (BLR).

A question arises: can we identify periodic variations? Such periodic variability should correspond to orbital motion exclusively, while the other two processes could produce only 
quasi periodic signals. Having an estimate of the BH masses, we can easily filter expected domains of periodicity that could correspond to the orbital motion, for the optical spectral range, where we have the longest observing records. Unfortunately, the brightest active galactic nuclei were identified only about 70 years ago. Therefore observing records are relatively short, and do not allow to trace many orbits in historical light curves. Extensive spectroscopic observations started even later, because the brightest AGN were still faint for the instrumentation available till the mid 1970s. With such limitation in the data, it is really hard to prove that the signal is periodic. If we were able to reveal periodic signals on the orbital timescales, combining results from the light crossing timescales, and measuring the broad emission line shapes (since we know that the low-ionization part of the emitting region is virialized) we could really be able to map the BLR.

\section{Light curve variability patterns}

AGN variability patterns present in their light curves mainly correspond to red noise, giving spuriously high significance levels to low frequency periods (see Westman et al. 2011, Vaughan et al. 2016 and Bon et al. 2016).

AGN have been since long known to be dominated by red noise-like light curves. Therefore, standard methods, such as the Lomb-Scargle (LS) method (Lomb 1976, Scargle 1982) may show very high peaks but the probability of a false positive computed with conventional statistical approximations may not be valid. Such problems in using LS periodogram tests for the analysis of AGN X-ray light curves has been known for some time, and they appear especially serious for very frequent quasi-periodic variability detections over long time-scales which turned out to be false (e.g., see discussion in Vangham 2006).

AGN light curves can be modeled as damped random walks (DRW) or with autoregressive models (as it has been known since many years; e.g., Fahlman \& Ulrych 1975, Gaskell \& Peterson 1987). There are some attempts to systematically search for periods in quasars LCs taking this problem into account (e.g. see Charsi et al. 2016, Liu et al. 2016). These studies show that periodicities of some candidates show very significant power-spectrum peaks compared to expectation for red noise. In some cases there is evidence of even more then one period with very high significance compared to autoregressive (AR) expectations (see e.g. Bahta et al. 2016).

Nevertheless, even though activity within one period might look stochastic compared to the DRW tests, repeating patterns could appear on very long light curves, showing the same complex patterns starting to repeat themselves after some periodic interval (see folded light curve presented in Fig. 1, where one can see that noisy pattern repeated after 5676 days in the NGC 5548 optical light curve; more details are available in Bon et al. 2016). Periodicity was found using a new method, similar to phase dispersion minimization (PDM, see Stellingwerf 1978), specially developed for the uneven-sampled 43 yr data series of NGC 5548 (Bon et al. 2016). Beside light curves, radial velocity curves of NGC 5548 measured on the broad $\mathrm{H} \beta$ profile show periodicity of $5550 \mathrm{~d}$, with a peak above 95\% AR significance level (of LS false alarm probability), as shown in Fig. 2, (see Table 3 in Bon et al. 2016). Using a PDM method and the new compiled light curve from Fig. 1 we found significant peak at $5678 \mathrm{~d}$.

Until now there are only few candidates with the detection of the same periodicity in both light and radial velocity curves: e. g., NGC 4151 (Bon et al. 2012) and NGC 5548 (Li et al. 2016, Bon et al. 2016). Both candidates have been monitored for very long time intervals, with probably the longest and best sampled data series of spectra and light curves, spanning over 43 years for NGC5548 and over 25 yr for NGC4151. 


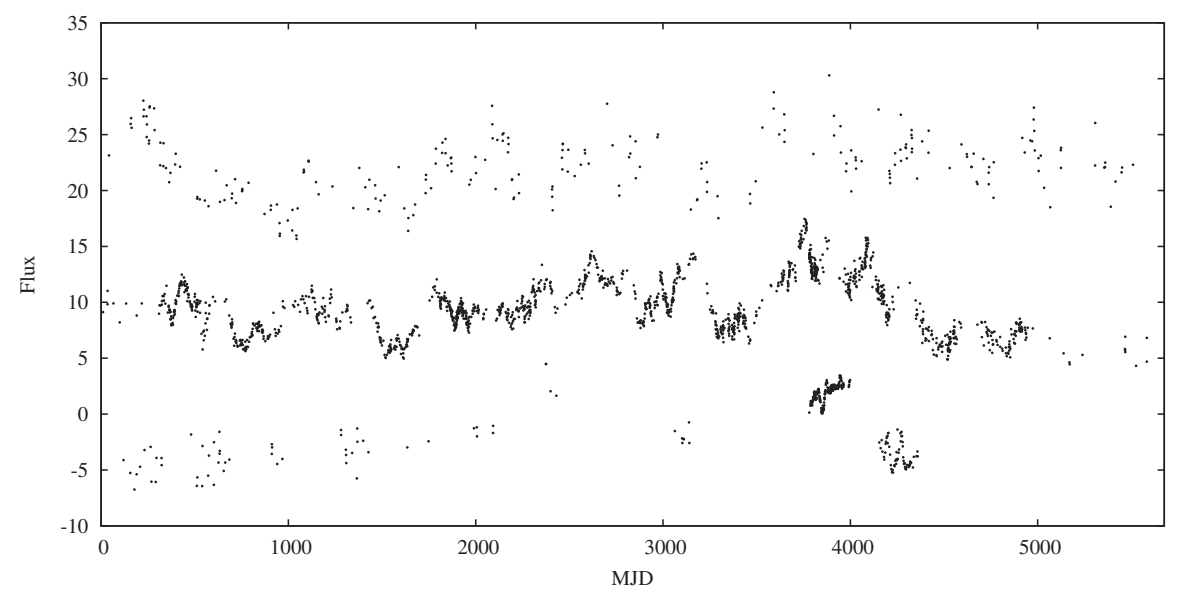

Figure 1. Folded phased diagram of NGC 5548 continuum flux 43 yr long light curve, assuming 5676 days periodicity, here presented to show an example of repeating noisy pattern light curve. Top light curve is pre AGN watch epoch, middle is AGN watch epoch Sergeev et al. (2007), and bottom is post AGN watch epoch constructed from new observations presented in Bon et al. (2016), Lu et al. (2016), Pei et al. (2017).

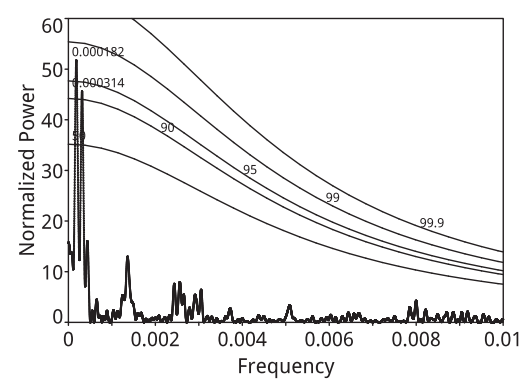

Figure 2. Example of Lomb-Scargle periodogram of NGC 5548 radial velocity curve constructed from the red side broad $\mathrm{H} \beta$ half-widths measured at $75 \%$ of maximum (see Bon et al. 2016). Red noise background spectrum significance levels are marked.

\section{Physical interpretations}

Probably the most plausible scenario for relatively short periodicity is orbital motion (see eg. Bon et al. 2012, 2016; Graham et al. 2015a, 2015b). Some mechanisms, are geodetic precession (Begelman et al. 1980), accretion disk precession due to a second black hole (Katz 1997), and disk-self warping induced by radiation pressure (Pringle 1996). Some of these periodic AGN are recognized as SMBBH candidates, like NGC4151 (Bon et al. 2012), OJ287 (e.g. Pihajoki 2016), PG1302-102 (Graham et al. 2015a). Geodetic precession is excluded since it occurs on time scales that are in general much longer than currently available monitoring times. Periodicity could be due to the presence of a longlived hotspot in the disk (Jovanović et al. 2010). We note however that the flare lasted only about a year, and was spiraling outwards in the model of Jovanović et al. (2010). Therefore, it would be more likely that the hotspot could be periodically generated by an orbiting body passing through the accretion disk (eg. Kieffer \& Bogdanovic 2016, Pihajoki 2016, Bon et al. 2016), with an orbital period similar to S0 stars in our Galaxy, or alternatively, a periodic enhancement of the inner part of the disk producing the X-ray emission (Fausnaugh et al. 2015, Bon et al. 2016). 
Graham et al. 2015b calculated that warped disks around single black holes are not favored in an AGN context unless very special conditions are satisfied. If the accretion disk is warped, it could lead to precession of the jet, but again with periodicity much longer then current monitored time. Yet another alternative possibility could be an obscuration of the central accretion disk, and of part of the BLR by a moving obscuring object (Bon et al. 2016), or moving emitting region, since BLR could consist of two different emitting components (Bon 2008, Bon et al. 2009a,2009b, 2015).

\section{References}

Begelman, M. C., Blandford, R. D., \& Rees, M. J. 1980, Nature , 287, 307

Bhatta, G., Zola, S., Stawarz, Ł., et al. 2016, Astrophys. J. , 832, 47

Bogdanović, T., S., Britton, D., Sigurdsson, S., \& Eracleous, M., 2008, ApJS, 174,455

Bogdanović, T., Eracleous, M., \& Sigurdsson, S. 2009, New Astronomy Reviews , 53, 113

Bogdanović, T. 2015, Astrophysics and Space Science Proceedings, 40, 103

Bon, E., 2008, SerAJ, 177, 9

Bon, E., Popović, L. Cr, Gavrilović, N., La Mura, G. , \& Mediavilla, E., 2009, Mon. Not. R. Astron. Soc. , 400, 924

Bon, E., Gavrilović, N., La Mura, G., \& Popović, L. Č., 2009, NewAR, 53, 121

Bon, E., Jovanović, P., Marziani, P., et al. 2012, ApJ, 759, 118

Bon, E., Zucker, S., Netzer, H., et al. 2016, ApJS, 225, 29

Bon, N., Bon, E., Marziani, P., \& Jovanović, P. 2015, Astrophysics and Space Science , 360, 7

Charisi, M., Bartos, I., Haiman, Z., et al. 2016, MNRAS, 463, 2145

Fausnaugh, M. M., Denney, K. D., Barth, A. J., et al. 2016, ApJ, 821, 56

Fahlman, G. G. \& Ulrych, T. J. 1975, ApJ, 201, 277

Gaskell, C. M. \& Peterson, B. M. 1987, ApJS, 65, 1

Gaskell, C. M. \& Klimek, E. S., 2003 Astronomical and Astrophysical Transactions, 22, 661

Gaskell, C. M.,2009, New Astronomy Reviews , 55, 140

Graham, M. J., Djorgovski, S. G., Stern, D., et al. 2015a, Nature, 518, 74

Graham, M. J., Djorgovski, S. G., Stern, D., et al. 2015b, MNRAS, 453, 1562

Jovanović, P., Popović, L. Č., Stalevski, M., \& Shapovalova, A. I. 2010, Astrophys. J. , 718, 168

Kieffer, T. F. \& Bogdanović, T. 2016, ApJ, 823, 155

Komossa, S. \& Zensus, J. A. 2016, Star Clusters and Black Holes in Galaxies across Cosmic Time, 312,13

Li, Y. R., Wang, J. M., Ho, L. C., et al. 2016, ApJ, 822, 4

Liu, T., Gezari, S., Burgett, W., et al. 2016 ApJ, 833, 6

Lomb, N. R., 1976, Astrophysics and Space Science, 39, 447

Lu, K.-X., Du, P., Hu, C., et al. 2016, Astrophys. J. , 827, 118

MacFadyen, A. I. \& Milosavljević, M. 2008, ApJ, 672, 83

Netzer, H. 2013, The Physics and Evolution of Active Galactic Nuclei Cambridge, UK: Cambridge University Press

Pei, L., Fausnaugh, M. M., Barth, A. J., et al. 2017, arXiv:1702.01177

Pihajoki, P. 2016, MNRAS, 457, 1145

Plavchan, P., Jura, M., Kirkpatrick, J. D., Cutri, R. M., \& Gallagher, S. C. 2008, Astrophysical Journal Supplement , 175, 191-228

\&Pringle, J. E. 1996, MNRAS, 281, 357

Scargle, J. D., 1982, ApJ, 263, 835

Sergeev, S. G., Doroshenko, V. T., Dzyuba, S. A., et al. 2007, Astrophys. J. , 668, 708

Stellingwerf, R. F. 1978, ApJ, 224, 953

Vaughan, S. \& Uttley, P. 2006, Advances in Space Research, 38, 1405

Vaughan, S., Uttley, P., Markowitz, A. G., et al. 2016, MNRAS, 461, 3145

Wilson, A. S. \& Colbert, E. J. M. 1995, ApJ, 438, 62 